\title{
A Model of Plasma Sheets in Equilibrium
}

\author{
S. Koutchmy
}

Institut d'Astrophysique de Paris-CNRS, 98 Bis Bd Arago, F-75014 France

M. Molodensky

IZMIRAN, Moscow region, Troitsk 142192 Russia

D. Vibert

Laboratoire d'Astronomie Spatiale, BP 8, F-13376 Cedex 12, Marseille France

\begin{abstract}
We consider large coronal streamers as confined thin plasma sheets overlying the filament and/or the magnetic polarity inversion lines. Confirmation is found in recent synoptic LASCO/SOHO data and simulations. We discuss the $3 \mathrm{D}$ axi-symmetric consistent MHD solution which provides the necessary properties of confinement and equilibrium by including gravity forces in the Grad-Shafranov equation.
\end{abstract}

\section{Introduction}

It has been known for a long time (Lockyer 1931, Vsekhsvjatsky 1963) that a connection exists between filaments and large coronal streamers. Both good resolution photometric data and a simple magnetic structure have been worked out by Koutchmy (1971) to interpret eclipse data and later, balloon data by Bohlin and Garrison (1974). Radially extended blades or sheets overlying the magnetic polarity inversion line (MPIL) were considered. Projection effects are of fundamental importance in interpreting white light (WL) images, so numerical models greatly help in removing ambiguities. Independently, resistive MHD models with increased sophistication have been proposed to explain the behavior of coronal streamers, especially when the physics of CMEs is considered. Among the latest work that follow the lines originally proposed by Pneuman and Kopp, we note the papers by Mikic and Linker (1994) and by Wu, Guo and Dryer (1997). Usually it is implicitely assumed that the WL coronal structures exactly match magnetic field lines computed by the model. However, from the analysis of prominence fine structures at least, this is not the case. We have the same difficulty when dealing with WL fine loop structures. In this short contribution we do not imply plasma structures are identical to magnetic lines but rather consider the confinement of these structures as well as the equilibrium of the coronal plasma (against gravity) at large scales, without considering the dynamical aspects. 


\section{Observations}

a) Many high-quality eclipse images show convincingly the direct links between prominences and coronal structures. Their 3D geometry is usually not easy to guess. Stereoscopic viewing helps identification (see Koutchmy and Molodensky 1992, Molodensky et al. 1996).

b) An extended new data set is available after the first year of operation of the SOHO mission. WL images of the corona are now collected at a high rate and good resolution is routinely achieved. We selected images taken during one solar rotation to look at the corona from different points of view, assuming rigid rotation. Images were taken at the time of the deep solar minimum in 1996, from April 11 to 28 . The corona was particularly quiet, showing sometimes a very narrow equatorial sheet, at least on one side of the limb. A model was designed to represent the rather narrow distribution of plasma densities across the sheet (Vibert 1997). The shape of the sheet was taken as a radial extension of the MPIL at $r=2.5$, computed using the potential field approximation and the available photospheric magnetographic data. Integration along the line of sight was performed taking into account the 3D geometry. Representative results of both the observed coronal structures and of the close agreement in the images given by the model, were shown at the meeting and can be found, e.g., in Vibert (1997). Even more convincing is the movie assembled using this material.

c) It is clear that this simple model confirms the importance of geometrical effects including fold-like configurations and the sheet-like nature of the streamer belt. A definite density enhancement occurs inside the sheet defined by the MPIL. This confinement of the plasma in a narrow sheet is quite similar to what is known to occur in the case of a large prominence ('curtain-type'). Accordingly, we developed an analytical model partly inspired by the classical model of prominences by Kippenhahn and Schluter (1957).

\section{Theoretical Modeling}

We make several assumptions: axisymmetry, negligible dynamical effects, and a simple magnetic configuration. We looked further at fully consistant MHD solutions of the well known Grad-Shafranov equation (Landau et al. 1984) where forces due to gravity are taken into account. The parameters are chosen in such a way to guarantee that the plasma density in the equatorial sheet well exceeds the density of the surrounding corona. The plasma temperature can be determined from the equilibrium equations determining the pressure and density. The measured temperature of the corona sets stringent constraints on the free parameters. The configuration of the resultant magnetic field is then different from that proposed by Pneuman and Kopp (1971) and Alexeev et al. (1982). The equilibrium configuration in spherical coordinates of a plasma in a magnetic and gravitational field can be written as:

$$
\nabla p-\frac{j^{*}}{c r \sin \theta} \nabla \Psi+\rho \nabla \Phi=0
$$


where $\Phi$ is the gravitational potential and $\Psi$ is the flux function of the magnetic field satisfying the following relation:

$$
\Delta^{*} \Psi+I I^{\prime}(\Psi)=\frac{4 \pi}{c} j^{*} r \sin \theta
$$

where

$$
\Delta^{*} \Psi=\frac{\partial^{2} \Psi}{\partial r^{2}}+\frac{\sin \theta}{r^{2}} \frac{\partial}{\partial \theta}\left(\frac{1}{\sin \theta} \frac{\partial \Psi}{\partial \theta}\right)
$$

The variable $j^{*}$ denotes that electric currents may exist besides $j_{\phi}$. When $\Phi=0$, eq. (2) reduces to the well known equation of Grad-Shafranov for the function $\Psi$.

Details of the treatment and the discussion of different solutions that we have obtained will be given in a more extended forthcoming paper. Figure 1 is an illustration of the set of magnetic field lines we obtained for the two cases which are deduced, depending of the thickness of the sheet we want to have: i) at left is the classical solution of the Pneuman and Kopp type which is coming from this treatment; ii) at right is the new solution we propose here that gives a well confined thin equatorial sheet.
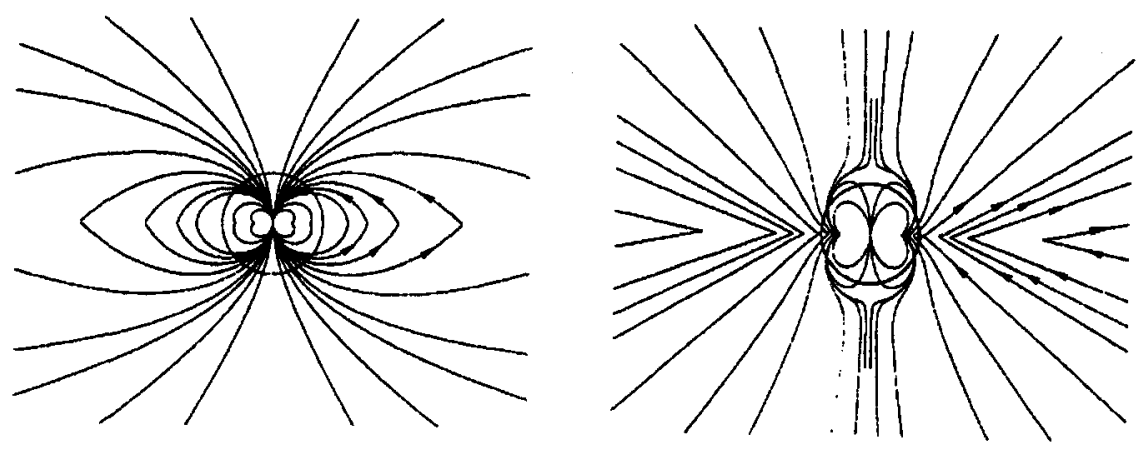

Figure 1. The set of magnetic field lines corresponding to the two solutions we deduced to describe the equatorial plasma sheet in equilibrium. Only the solution at right gives a well confined thin sheei.

\section{Conclusions}

a) The narrow distribution of densities inside an equatorial sheet can be in equilibrium provided the coronal magnetic field corresponds to the solution we show at right on Figure 1 (we call it 'concave current sheet'). Note that the classical solution of the Kopp-Pneuman type ('convex current sheet') cannot provide such confinement and would evacuate the region of the equatorial sheet, where the cusp forms.

b) A narrow heliospheric sheet is observed in the interplanetary medium out to very large distances as illustrated by the Ulysses results. We can then speculate 
on the rather minor effect that the dynamics (wind) would play in our model.

c) Our model predicts some peculiar structures inside the equatorial sheet: magnetic lines across it show a concave shape. Features like this are now well observed in the equatorial flow of the wind seen on SOHO/LASCO movies.

d) A quite similar magnetic structure was calculated by Uchida and Low (1981) for the magnetosphere of a star with an accreted magnetized mass. We, however, noticed a fundamental difference with what we are proposing: in the case of an accretion disk, a uniform outer magnetic field is postulated. We do not have such a constraint in our model.

e) Finally, it could be useful to look more carefully at the magnetic structure of polar regions where prominences are usually absent. We plan to work soon on that.

\section{References}

Alexeev, I. I., Kropotkin, A. P. and Veselovsky, I. S. 1982, Solar Phys., 79, 385 Bohlin, J.D. and Garisson, L.M. 1974, Solar Phys. 38, 165

Kippenhahn, R. and Schlüter, A. 1957, Zeit. für Astrophys., 43, 36

Koutchmy, S. 1971, A\&A, 13, 79

Koutchmy, S. and Molodensky, M.M. 1992, Nature, 360, 717

Molodensky, M. M., Starkova, L. I., Koutchmy, S. and Ershov, A. V. 1996, in Solar Drivers of Interplanetary and Terrestrial Disturbances, (eds.) Balasubramaniam et al., A.S.P. Conf. Ser. Vol. 95, San Francisco, p. 385

Landau, L. D., Lifshits E. M. and Pitaevskii, L. P. 1984, Electrodynamics of Continuous Media, 2nd edition, Translated by J. B. Sykes, J. S. Bell and M. J. Kearsley, Pergamon Press, Oxford

Lockyer, W.J.S. 1931, MNRAS, 41, 7, 801

Mikic, Z. and Linker, J.A. 1994, ApJ. 430, 898

Pneuman, G. W. and Kopp, R. A. 1971, Solar Phys. 18, 258

Uchida, Y. and Low, B.C. 1981, J. Astrophys. Astr. 2, 405

Vibert, D. 1997, These Dr es Sc., Marseille Univ., St Charles, France

Vsekhsvjatsky, S.K. 1963, in The Solar Corona, (ed.) J.W. Evans, Academic, San Diego, p. 271

Wu, S.-T., Guo, W. P. and Dryer M. 1997, Solar Phys., 170, 265 